口-

The combination of several non-guaranteed random energy sources (RES), conventional sources, and nonconstant consumer loads in a local system leads to stochastic power imbalances. This study objective consists in determining the possibilities of ensuring the power balance in a hybrid power generation system with a standby generator and a search for the methods of calculating the optimal parameters to achieve energy balance. This objective is achieved by simulating the processes inherent in wind and solar power engineering and the regimes of energy consumption through a combination of random functions with a standard probability distribution. Aggregated data on weather factors for several years in a region with a high renewable energy potential which can be used to describe the behavior of wind and solar energy over time were used as experimental data. The use of multiple simulations of random processes with calculated parameters has made it possible to draze conclusions about the presence of certain ratios of power and the generator control modes. These ratios can determine minimum energy and consumption losses, reduce the likelihood of energy imbalance, more efficiently use the reserved power. Specific features of the stochastic nature of RES related to the presence of trends and random fluctuations at short hourly intervals were additionally taken into account. Possibilities of varying the conditions of and switching on and off of the standby generator were provided. The existence of some ranges was established for the installed power of the generator outside which its use becomes inefficient. The proposed approach makes it possible to find the probability of various system states, assess the reliability of energy supply, and minimize unproductive losses

Keywords: local power system, renewable energy sources, diesel generator, power balance
DOI: $10.15587 / 1729-4061.2021 .245557$

\section{ENSURING POWER BALANCE IN A HYBRID POWER SYSTEM WITH A STANDBY GENERATOR}

Mykola Kuznietsov

Doctor of Technical Sciences, Senior Researcher Department of Integrated Energy Systems Institute of Renewable Energy of the National Academy of Sciences of Ukraine Hnata Khotkevycha str., 20-a, Kyiv, Ukraine, 02094

O Iga Lysenko Corresponding author Doctor of Technical Sciences, Professor*

E-mail: helga_vl@ukr.net

Andri Chebanov $\mathrm{PhD}$, Associate Professor*

D m y t ro Zhuravel

Doctor of Technical Sciences, Professor Department of Technical Service in Agriculture**

*Department of Power Engineering and Automation**

**Dmytro Motornyi Tavria State

Agrotechnological University

B. Khmelnytsky ave., 18, Melitopol, Ukraine, 72312
Received date 28.10.2021 Accepted date 03.12.2021 Published date 24.12.2021
How to Cite: Kuznetsov, M., Lysenko, O., Chebanov, A., Zhuravel, D. (2021). Ensuring power balance in a hybrid power system with a backup generator. Eastern-European Journal of Enterprise Technologies, 6 (8 (114)), 6-15. doi: https://doi.org/10.15587/1729-4061.2021.245557

\section{Introduction}

Most of the problems related to the large-scale introduction of renewable energy sources (RES) are related to the uneven regime of their generation. When integrating powerful wind power plants (WPP) and solar power plants (SPP) in a general net, their impact is mitigated due to the total capacity of the power system. At the same time, it would be appropriate to consider independent use of scattered RES and those situated close to final consumers in combination with conventional sources. In this case, a group of WPP or SPP together with consumers can be considered as a local energy system (LES). It can be defined as a set of generating electrical equipment of limited power, converters, and consumers of electricity connected in a distribution net where overall electromagnetic processes take place [1]. In this case, it is necessary to consider the interchangeable power generation by wind and solar power plants and energy consumption as a united random process. The share of energy consumed from a united power system decreases, however, requirements to balance the local net grow.

The growing share of wind and solar generation and the formation of local systems have caused a number of new problems because of non-guaranteed generation. A need to develop mathematical approaches that will adequately describe random processes in hybrid power systems has appeared. There are various solutions for concrete systems, however, the development of more unified probabilistic models remains relevant. 


\section{Literature review and problem statement}

The problem of optimal organization of limited power systems with a significant RES share has been widely reflected in studies. The number of studies has grown, especially when the cost of the technologies based on renewable energy sources (especially photovoltaics) has reduced significantly and the existing problems still required their solution in conventional energy arousing interest in the construction of autonomous and hybrid power systems. An overview of studies on the stability of autonomous power systems using RES depending on their configuration is given in [2]. An extensive overview of hybrid system configurations, methods of mathematical modeling, and control strategies are considered in [3]. It is shown that the combined use of different renewable energy sources as an alternative to extracted fuels is increasing. Such energy systems are considered hybrid systems. It is noted that hybrid energy systems can be a viable alternative to electric nets or the use of conventional fuels in remote areas around the world. It is shown in [4] that systems with multiple energy sources such as sun and wind and centralized nets have the best results in terms of reliability and cost. It is noted that a preliminary review and forecast of both local climatic conditions and features of energy demand are of high importance. Therefore, the use of wind and solar energy in hybrid systems is recognized as a promising and urgent task. It is important to take into account local conditions to achieve reliable supply. This requires studies on how such reliability can be ensured. A significant place among the studies is occupied by the topic of energy supply to remote or isolated objects, objects with difficult access to electric nets [5]. The importance of forecasting loading regimes in such systems is emphasized. There are issues of forecast quality for individual objects that need studies. Generators with internal combustion engines (for example, diesel generators) are mostly considered as conventional energy sources. It is often noted that their use creates a number of problems including environmental ones. It is shown in [6] that wind or solar power engineering helps solve them partially. This is an international trend towards the promotion of renewable energy sources as a part of energy safety and reduction of greenhouse gas emissions. Therefore, there is a problem of optimizing the modes of fuel use to reduce the negative impact which lessens the RES benefits. Attention is focused in [7] on the current forecasting of short-term changes inherent in renewable energy which requires special methods for both actual forecast and study of its impact on the reliability of power supply. The available studies also address regional specificities. For example, various hybrid configurations of wind, solar, and diesel systems for remote settlements are presented in [8]. Various configurations are considered on an example of the Arabian region rich in RES: only diesel fuel, wind-diesel generation, solar energy and diesel fuel, and a combination of the mentioned sources. Local wind velocity, solar radiation intensity, and hourly averaged loads were used as input data. In [9], the analyzed object is located far away from the net, so, the use of renewable energy sources predominates. Technical and economic analysis of the system has shown that the system has a renewable energy utilization rate of $65 \%$ and low power generation costs. However, such results are specific to the studied regions and it is desirable to have a common method or mathematical model for their application in different locations.
An example of the use of wind and solar energy as a supplement to conventional energy sources is given in [10]. Diesel generators have been added to ensure uninterrupted supply because of the intermittent nature of renewable energy sources. For isolated consumers powered from autonomous hybrid systems based on solar and wind generation [11], possibilities of ensuring the reliability of supply with a support of a diesel generator have been investigated. Various types of power generation are considered and it is shown that the system efficiency is higher at simultaneous use of various types of renewable sources and considerably reduced loading of the diesel generator. There is a need to find such a combination of different sources that would more fully meet the requirements of such a system. There are different computational methods for estimating the behavior of RES power systems depending on conditions at the object of study. For example, optimization of the diesel generator operation in real time is considered in [12]. Some studies use computation means, such as HOMER and MATLAB [13], to find optimal system configuration using various target functions. It was noted in [14] that hybrid systems have a better potential for use compared to the use of a single source, e. g. a diesel generator. A diesel generator is sometimes considered as an emergency standby power supply [15] in cases of low levels of renewable energy or action of unplanned loads. To solve this problem, appropriate mathematical models of a concrete hybrid system are required, e. g. the model considered in [16] concerns balancing by a hydroelectric station. Therefore, the method of optimization of the energy supply structure should be based on the chosen mathematical model which would meet specific conditions of the optimization problem.

Thus, a general range of issues to be considered when planning a local power system was outlined: choice of renewable energy sources, means of regulating the power balance, taking into account climatic conditions, forecasting, application of methods of system modeling, and assessment of results. However, the studies considered concern mainly the economic component and sometimes the environmental component which requires integrated assessments. Instead, the reliability of power supply should be assessed based on probabilistic assessment of processes that are random in their nature and relatively rapidly (within a few minutes) change their parameters. Ignoring such properties reduces the reliability of the results obtained. Obtaining an appropriate estimate of the efficiency of the chosen energy supply system requires a special mathematical model that takes into account specifics of the stochastic nature of the processes occurring in power generation and consumption. It is important to take into account both local climatic conditions and characteristics of energy demand.

This study features taking into account specific behavior in time of wind and solar energy sources and the possibility of obtaining probabilistic estimates with an admissible error.

\section{The aim and objectives of the study}

The study objective is to determine the possibilities for ensuring the energy balance in a hybrid power system with a standby generator and to search for methods to calculate the optimal parameters in order to achieve the energy balance. This will make it possible (as early as at the stage of designing the local power system and organizing its operation) to select a configuration of generating capacity and operating 
conditions of standby generators in which load drops and/or excess electricity will be minimal. It will also enable determining the probability of undesirable states of the system to assess the risk of energy supply.

To achieve this objective, the following tasks were set:

- to formulate a problem of stochastic optimization of structure and modes of operation of a hybrid power system containing non-guaranteed and controlled power generating sources;

- to offer actuation conditions and operating modes of a standby power supply source (electric generator);

- to choose a method of modeling random processes of power generation and consumption;

- to analyze features of ensuring power balance depending on parameters of standby power generation and identify possibilities of ensuring the required power balance.

\section{The study materials and methods}

The wind-diesel power stations that supply energy (heat and electricity) to free-standing facilities can be considered well-studied examples of hybrid LES with a fuel component. Most often, such wind-diesel systems contain the following components: one or more wind turbines, one or more diesel plants, and a consumer load. In a more general case, ballast (damping) loads or a secondary consumer, an energy accumulating system, and consumption control elements can also be used. Such options can be described in the framework of the proposed model and their impact is the subject of further studies. In this case, energy can be distributed among consumers during a day according to a specified program but can also be random. As a rule, it is an agreed graph with current deviations (fluctuations) of different volatility. In conventional systems of this type, the main task of RES consisted in reducing the consumption of fuel and engine life, i. e. mainly a supporting role. In modern RES-diesel hybrid systems, a diesel generator can act as an auxiliary source which makes it possible to avoid significant power imbalances.

Estimation of parameters of the power supply system operation by means of a mathematical model should give the chance to choose the best configuration, i. e. structure of generating supply capacities and regimes of their operation. In the most general case, to optimize the system operation which depends on random weather factors, it is necessary to formulate a convex problem of parameter optimization or optimal control using controlled power generation. Next, it is necessary to find a solution for each individual instantiation of a random process as a deterministic function (direct method) and then perform statistical processing of the results of multiple process simulations. Another way consists in constructing the distribution density functions of the desired objective function with the use of a set of random instantiations of the process followed by estimation of expected values and their confidence intervals. A parametric approach is possible in this case: estimation of distribution indicators by the Monte Carlo method or direct processing of possible results.

Most problems of optimizing the composition and regimes of operation of the power generating system cannot be described by the criterion of optimality alone. A heuristic approach is appropriate in this case, for example, assessment of various indicators and choice of an optimal value depending on the conditions and requirements of the power generating system. Parameters of RES generation in the proposed model are calculated based on actual long-term data on weather factors (wind velocity and solar radiation level). With regard to energy consumption, it was assumed that the schedule of energy consumption can be predicted with some accuracy. To do this, a forecast of the expected RES capacity is used: this can be daily forecasts or long-term averaged data for a particular period of a year (usually for a month). The difference between the RES capacity and the expected load is compensated by the planned operation of conventional sources, such as the electric net (Fig. 1). Such a hybrid system can contain solar and wind power plants as a source of unguaranteed power and an additional controlled electric generator. This system has to be able to connect to the net according to a pre-declared schedule of energy consumption. A local system of this type usually includes a consumer of limited capacity (up to $1 \mathrm{MW}$ ) and the total RES capacity should be commensurate to the load capacity taking into account the efficiency of the installed RES capacity.

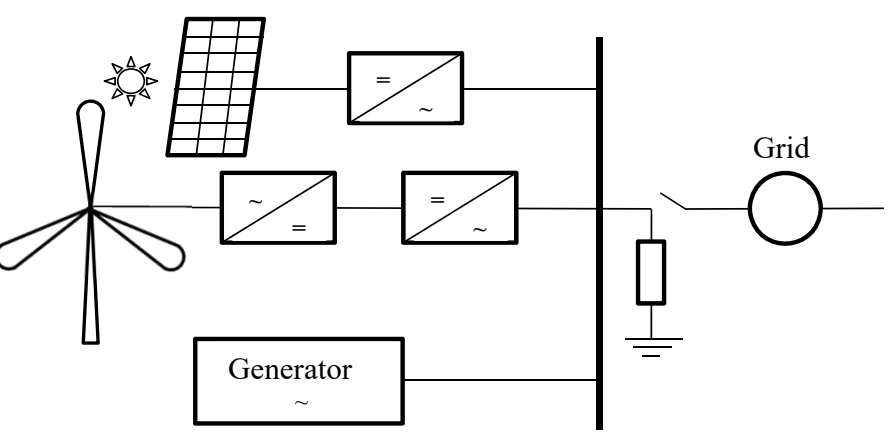

Fig. 1. A hybrid system with renewable energy sources and a controlled generator

The Law of Ukraine on Electricity Market provides for market participants using the RES the availability of forecast daily and hourly schedules of expected generation and deviation from the stated schedule are considered an imbalance having economic consequences. In the case of an autonomous system, consumption restrictions will be the natural result of imbalance. The study subject is the random component of the power balance which can be defined as the difference between the planned and actual schedules of generation and consumption. It is assumed that the system operation modes are controlled with a certain step using averaged values in a unit time interval $\Delta t$. This component can be described by the following equation:

$$
p_{i j}=\left[\left(w_{i j}-w_{i}\right)+\left(s_{i j}-s_{i}\right)\right]-\left(u_{i j}-u_{i}\right),
$$

where $u_{x}$ is the level of electricity consumption (load);

$\omega_{x}$ and $s_{x}$ are powers of WPP and SPP, respectively;

$i$ is time index (step number or $t_{i}$ );

$j$ is the day number.

Here, $p_{i j}$ is the deviation from the load schedule and $u_{i j}$ is the level of electricity consumption on a given day and time. Indicators with one index are averages over a certain time of day (daily course), in particular, $u_{i}$ should correspond to the planned schedule of consumption. Then $p_{i}=\left(w_{i}+s_{i}\right)-u_{i}$ 
corresponds to the planned generation, i. e. it is provided from the net or other sources. It is more convenient to normalize all values as a normalizing factor considering, e. g. the maximum loading. Then all facilities are taken into account as relative values. Expression (1) describes the current power imbalance with positive values indicating the excess generation.

The following problem was considered. Let the consumption be considered deterministic, equal to the average daily wind and solar generation, i. e. $u_{i}=w_{i}+s_{i}$.

This condition corresponds to the planned schedule for the forecast RES generation in the current day. Then the power imbalance is determined by random deviations of WPP and SPP from the average daily run and by similar deviations of the consumption level. The daily course of the power balance under such conditions can be considered an ergodic process. Since solar generation has a large variability during the day and power consumption is designed for averaged generation, it is during the day that most unbalanced states occur with a significant share of SPP while the WPP impact is more uniform. In the event that the balance becomes negative (insufficient generation), an additional source (a standby generator) playing the role of a control function can be connected to reduce the imbalance. The goal consists in reducing the load loss to a certain allowable level. It is assumed that the generator operation is subject to certain rules: it is actuated when generation is insufficient indeed and switched off when the negative imbalance is reliably eliminated, i. e. it has a certain time delay. Another condition: the generator operates with a certain fixed power, i. e. the schedule of its operation regime is a function that looks like a sequence of rectangles of the same height (for a noncontrolled generator). Then the generator operation can be considered as a flow of events of various durations.

Obviously, the extra operation of the generator is unprofitable, therefore, the task of optimizing the mode of additional generation consists in ensuring the minimum (or specified) level of allowable consumption losses (some insensitivity zone is usually allowed). Under such conditions, optimization of the power system is the problem of optimal control with phase constraints with a discontinuous control function. Optimal control in this case consists in selecting and implementing the best program of the generator operation to achieve the desired state of the controlled object (the power generating system).
In this problem statement, the equation of power imbalance can be represented as:

$$
V(t)=P_{W}(t)+P_{S}(t)+P_{G}(t)-P_{U}(t),
$$

where $P_{G}(t)$ is a piecewise continuous control function of stepwise form; $P_{U}(t)$ is a known load function (planned consumption schedule plus random deviations); $P_{W}(t)$ and $P_{S}(t)$ are current capacities of the WPP and SPP; $V(t)$ is the function of the system state. The mathematical model of the RES generation process is described in [17] and the nature of the distribution of the random component is described in [17]. An energy accumulator is a desirable option; however, it is not considered in this problem as the generator operation is the study subject.

Simulation modeling of daily instantiation of a random process (2) with a large (up to a thousand) number of instantiations was sufficient for the stability of statistical parameters. The algorithm of the simulation model is shown in Fig. 2.

Statistical processing of simulation results makes it possible to calculate parameters of the distribution of the process values as random variables, in particular, mean values and mean square deviations. A number of estimates were obtained by direct calculation with a sufficient number of instantiations.

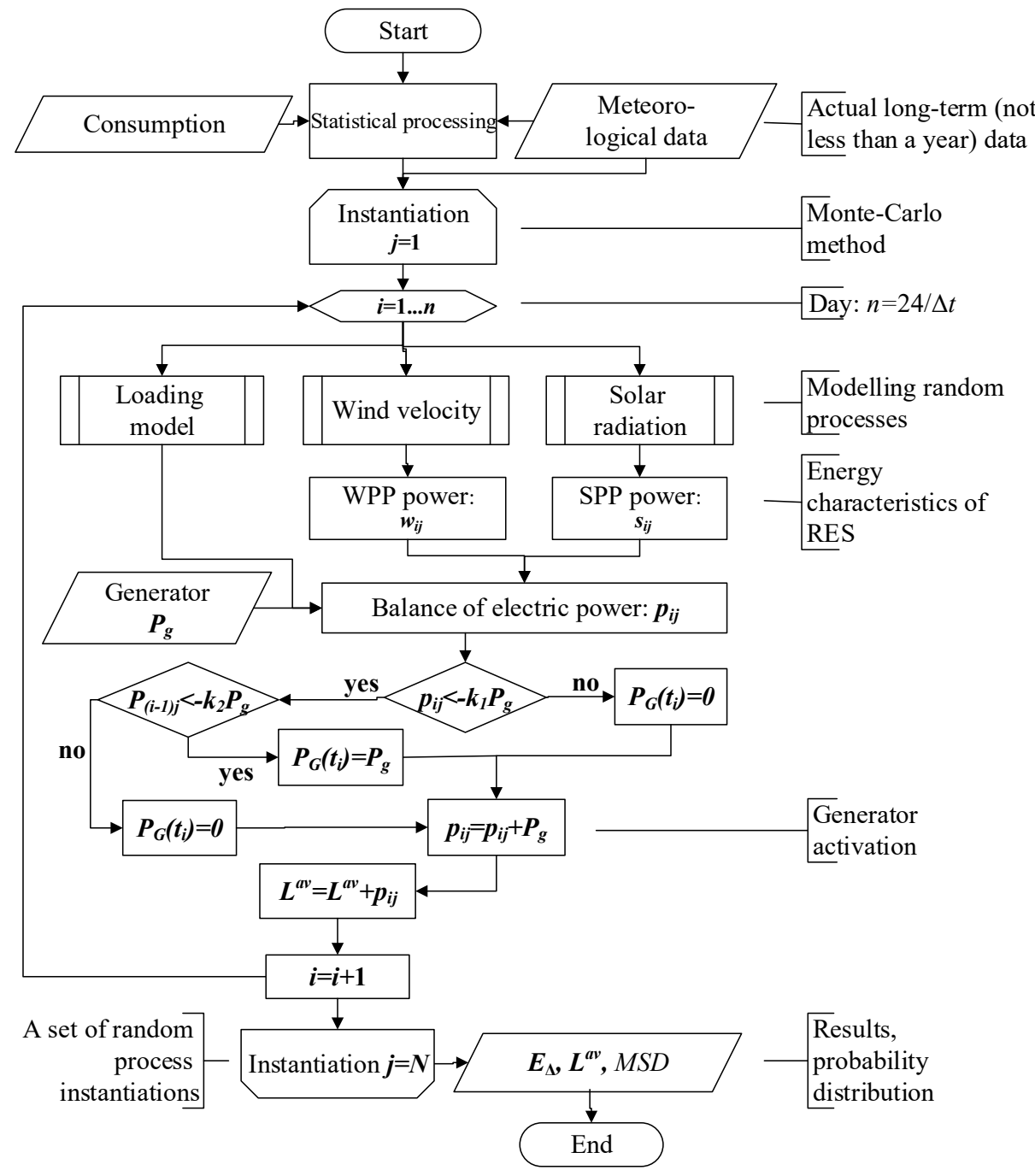

Fig. 2. Formation of energy balance with a standby generator 


\section{The results obtained in the studies of energy} balance parameters

\section{1. Statement of the problem of stochastic optimiza- tion of a hybrid power system}

To set the problem of optimal control, it is necessary to establish phase coordinates and equations of state. Current values of power imbalance can act as state variables (phase coordinates) and energy characteristics of WPP and SPP as state equations, i.e. the dependence of their power on wind velocity or solar radiation level, respectively.

In the classical problem of optimal control, we are talking about smooth functions. In this study, both generation and consumption (in the general case) are random functions, i.e. by definition, they are considered a discontinuous function at each point which makes it impossible to make a correct analytical decision. However, some estimates can be made for integrated indicators, in particular, the generated and consumed energy as cumulative power sums are described by continuous functions although their derivatives will be discontinuous. Therefore, it is advisable to consider the energy indicators of the power system which are also random processes but more predictable than the current powers. At this approach, the problem of optimal control can be expressed as follows:

$$
\begin{aligned}
& E_{V}\left(T, \alpha_{x}\right)=E_{R E S}\left(T, \alpha_{W}, \alpha_{S}\right)+ \\
& +E_{G}\left(T, \alpha_{G}\right)-E_{U}\left(T, \alpha_{U}\right) \rightarrow \min ,
\end{aligned}
$$

where $E_{x}\left(T, \alpha_{x}\right)=\int_{0}^{T} P_{x}\left(t, \alpha_{x}\right) \mathrm{d} t$ is energy as cumulative power; $\alpha_{x}$ are weight coefficients proportional to the corresponding rated powers; $E_{R E S}(t)=E_{W}(t)+E_{S}(t)$ is the RES energy.

The existence of the integral is ensured by the limited nature of the subintegral function which follows from physical conditions.

The dependences for the current power can be considered as equations of state:

$$
\partial / \partial t E_{x}\left(t, \alpha_{x}\right)=P_{x}\left(t, \alpha_{x}\right), \quad(x=W, S, U),
$$

with the following restrictions:

$$
\left.M\left\{E_{V}\left(t, \alpha_{x}\right)\right\}\right|_{T} \mid=0, \ldots, E_{V}\left(0, \alpha_{x}\right)=E_{V}\left(T, \alpha_{x}\right)=0,
$$

where $T$ is the cycle duration (usually a day or a month, depending on the forecasting possibility).

Terminal constraints (4) are determined by the assumption of a complete overall balance and daily cyclicity. Additional restrictions on individual components will be determined by their physical properties (rate of change, achievable capacity, etc.).

In formulation (3), (4) this is a Mayer problem with several phase parameters and fixed limits. Unlike the classical problem, the functions are not smooth here but at least continuous (energy as an integral, does not change abruptly in contrast to the current values of RES power).

Weight coefficients of the power balance components can be the parameters of optimization of LES operation (power selection and generator control settings). The behavior of individual components as random functions corresponds to the type of equipment, climatic conditions or season, geographical location, etc. The parts of renewable energy gener- ation (or wind and solar separately) act as phase coordinates and the power of the generator power as control.

Other possible optimization criteria: minimum of imbalance dispersion $D\left\{E_{V}(t)\right\}$, probability of getting in a certain interval $\operatorname{Prob}\left\{E_{\min }<E_{V}(t)<E_{\max }\right.$, minimum of positive imbalance (lost energy), negative imbalance (loss of consumption), minimum of the generator on-off cycles at admissible losses. The mathematical model assumes the possibility of finding these indicators.

The generator operation mode (time and duration of the switched-on state is determined by a random component, namely the current RES power and consumption. The state of the controlled system is also random and the change in its state caused by the generator impact can be considered a reaction to such influence. Thus, we have a dynamic system with random input data (WPP and SPP capacity and random consumption component), regulating external influence (generator on-off) and system response (change of the studied parameters). Direct study of random functions by analytical methods is difficult or impossible, so the indirect study methods, like simulation, seem to be appropriate to establish an implicit link between external influences and reaction.

Let us consider the following expressions as criteria for optimizing the LES control mode:

$$
L_{1}^{\min }=\max _{G} \min _{t} \int_{0}^{T}\left|V(t)^{-}\right| \mathrm{d} t,
$$

or

$$
L_{2}^{\min }=\min _{G} \int_{0}^{T}\left|V(t)^{-}\right| \mathrm{d} t,
$$

where $V(t)^{-}<0, \quad V(t)=V(t)^{-}+V(t)^{+}$are components of power balance (2) with different signs.

Here, $L_{1}^{\min }$ determines the lower limit of imbalance, and $L_{2}^{\min }$ determines the total loss of consumption. Under the conditions of forecasting, the condition of equality of average capacities of generation and loading is fulfilled:

$$
P_{U}^{a v}=\frac{1}{T} \int_{0}^{T} P_{U}(t) \mathrm{d} t=P_{R E S}^{a v}=\frac{1}{T} \int_{0}^{T}\left[P_{W}(t)+P_{S}(t)\right] \mathrm{d} t,
$$

where $T=24$ hours at daily forecasting or 1 month when using long-term data (at least a year).

\section{2. Operating conditions of the standby generator}

Let us assume that maintaining a negative balance at a certain level for a specified time is the condition of actuation (operation) of the generator in the system:

$$
P_{G}(t)=\left\{\begin{array}{l}
P_{g},\left[P_{R E S}(t)-P_{U}(t)>k_{1} P_{g}\right] \wedge \\
\left.\qquad \begin{array}{l}
P_{R E S}(t)+P_{R E S}(t-\Delta t)- \\
-P_{U}(t)-P_{U}(t-\Delta t)>k_{2} P_{g}
\end{array}\right], \\
0, \quad \text { other } \ldots
\end{array}\right.
$$

Here, the coefficients $k_{1}, k_{2}$ play the role of settings for controlling the generator operation mode when a certain level of imbalance of renewable power is the condition of its actuation compared to the generator power for the last few time intervals. If technical conditions of the generator oper- 
ation require a significant time delay, the control algorithm may provide for varying the duration of the interval $\Delta \mathrm{t}$ in expression (7).

A condition of a simultaneously set level of imbalance in the current and previous time intervals can be another option of settings for the generator actuation:

$$
\left[\begin{array}{l}
P_{R E S}(t)- \\
-P_{U}(t)>k_{1} P_{g}
\end{array}\right] \wedge\left[\begin{array}{l}
P_{R E S}(t-\Delta t)- \\
-P_{U}(t-\Delta t)>k_{2} P_{g}
\end{array}\right] \Rightarrow P_{G}(t)=P_{g}
$$

In contrast to (7), this condition implies a mandatory condition of imbalance in the previous moment. When regulating the sum of adjacent values, the condition can be provided by a significant excess of the permissible limit at the current time with a slight previous imbalance. However, in conditions of real variability of renewable sources, such significant imbalance jumps are unlikely. The dominance of solar energy in conditions of variable cloudiness may be an exception.

\section{3. Modeling the random processes of pow- er generation and consumption}

To describe the variability of power generation and consumption processes, observational data from real processes interpreted in terms of stochastic modeling were used [18]. Wind conditions are determined by the parameters of wind velocity distribution: mathematical expectation and dispersion (namely these indicators are recorded by the present-day meteorological instruments of the WPP). Parameters of Weibull distribution are often used as secondary indicators. According to them and the indicators of stability of wind velocities (in particular, duration of deviations of one sign can be considered with autocorrelation of the measurement time series), the probability of successive values of WPP power can be calculated [19, 20]:

$$
X\left(t_{k}\right)=X\left(t_{k-1}\right) e^{-\beta \Delta t}+\varepsilon \frac{\sigma}{\sqrt{2 \beta}} \sqrt{1-e^{-2 \beta \Delta t}},
$$

similarly, the SPP power can be described:

$$
z_{j}\left(t_{i}\right)=z_{j}\left(t_{i}\right)\left(1+C_{v} \varepsilon\right)+b\left(x_{i}-0.5\right) \leq Z\left(t_{i}\right)
$$

where $\beta$ and $\sigma$ are the parameters of drift and volatility of the process of changing the current WPP power; $C_{v}$ and $b$ describe the current variability of insolation; $z(t)$ and $Z(t)$ are the average and maximum achievable level for a given area and season. The complete idea of the current generation or consumption capacity is determined by the expression:

$$
P_{i}(t)=\omega(t)+\sigma_{D} \varepsilon+U(t)
$$

where $\omega$ is the trend curve according to the data of long-term observations (daily graph); $\sigma_{D}$ is the standard deviation of daily average values, as the forecast accuracy; $\varepsilon$ is the standard normally distributed random variable. The current fluctuations $U(t)$ are determined by expressions of type (9).

Due to the predefined parameters of these processes, properties of the input independent random process of power balancing can be described. The load level is previously defined as deterministic but without limiting the generality, it can have a random component distributed according to the normal law and with a variance characteristic of certain consumers. The process of regulating $P_{G}(t)$ as an external influence depends on the input process. With a given control algorithm, this process will be a deterministic function of a random variable, i.e. the results are also random variables. In the future, the processes of form (8) and (9) can be modeled using a generator of random numbers by forming daily instantiations.

\section{4. Features of ensuring the power balance}

The results of simulation at different values of generator power and in the presence of daily forecasting of the system are given in Table 1 (assuming that $k_{1}=1, k_{2}=2$ ) and shown in Fig. 3. As analysis of the system operation at different values of the rated generator power $P_{g}$ shows, a small power may not provide reliable elimination of imbalance and too high power leads to a decrease in the switch-on cases.

Table 1

Average value and MSD of negative balance at $k_{1}=1, k_{2}=2$, daily forecast (in relative units) 
minimal was noticeable at $P_{g} \approx 0.12$ (r. u.). Values of $P_{g}<0.15$ can be considered small and $P_{g}>0.5$ are large in this system.

In absence of daily forecasts, i. e. the second component in expression (10), the average level of consumption is assumed to be constant and equal to the average monthly capacity of RES without adjustment for daily deviations. Under such conditions, the balancing process will no longer be ergodic during the day requiring more instantiations to achieve a statistically stable result. The need for the regulating power of the generator is much higher but the behavior of the imbalance is qualitatively similar indicating the presence of an optimal value, almost twice as large (Table 2, Fig. 4).

Table 2

Average value and MSD of negative imbalance at $k_{1}=1$, $k_{2}=2$, monthly forecast (r. u.)

\begin{tabular}{|c|c|c|c|c|c|c|c|c|c|c|}
\hline$P_{g}$ & 0.0 & 0.05 & 0.1 & 0.15 & 0.2 & 0.25 & 0.3 & 035 & 0.4 & 0.45 \\
\hline$L_{2}^{a v}$ & 0.140 & 0.117 & 0.094 & 0.084 & 0.077 & 0.074 & 0.082 & 0.099 & 0.117 & 0.124 \\
\hline$\sigma_{2}$ & 0.165 & 0.149 & 0.124 & 0.108 & 0.098 & 0.094 & 0.107 & 0.122 & 0.138 & 0.144 \\
\hline
\end{tabular}

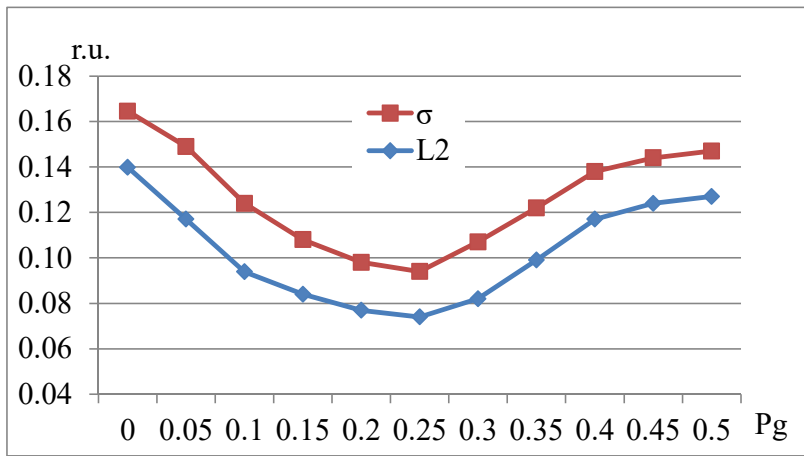

Fig. 4. Negative power imbalance, monthly forecast

As can be seen from Fig. 4, the minimum of the average imbalance coincides with the minimum scatter of $\sigma$ values (optimal value of $P_{g} \approx 0.24$ r. u.).

Fig. 5 shows an example of one of the daily instantiations of the random process of capacity balancing ( $b a l$ RES). The total daily balance is zero but there are significant values of negative balance during the day (from the $51^{\text {st }}$ to the $95^{\text {th }}$ 10 -minute interval). Partial imbalance compensation occurs due to the generator (gen) and as a result, the share of load loss $\left(L_{2}\right)$ decreases. The average power of the generator close to the optimal in terms of losses was considered. The selected control algorithm (7) allows the facts of short-term generator actuation which negatively affects its operation.
Changing the quantitative constraints in algorithm (7) leads to other values of average load loss and optimal power of the generator (Table 3 ).

Reduction of the threshold limits makes it possible to more accurately select conditions of the generator actuation and, respectively, reduce the negative imbalance. Reduction of load losses with additional actuation of the control generator must be accompanied by a certain increase in excess energy, i. e. generation losses. However, the generator effect on the level of energy loss is significantly less than on prevention of load losses (Fig. 6). Numerical imbalances can vary with different generator control algorithms but the qualitative relationship remains.

Table 3

Average negative imbalance $L_{2}^{a v}$, daily forecast (r. u.)

\begin{tabular}{|c|c|c|c|c|c|c|c|}
\hline \multirow{2}{*}{$k_{1}$} & \multirow{2}{*}{$k_{2}$} & \multicolumn{7}{|c|}{$P_{g}$} \\
\cline { 3 - 8 } & & 0.05 & 0.1 & 0.15 & 0.2 & 0.25 & 0.3 \\
\hline \multirow{2}{*}{1} & 1.5 & 0.054 & 0.045 & 0.045 & 0.052 & 0.059 & 0.062 \\
\cline { 2 - 8 } & 1 & 0.052 & 0.043 & 0.044 & 0.051 & 0.056 & 0.062 \\
\hline \multirow{2}{*}{0.5} & 1 & 0.052 & 0.038 & 0.029 & 0.028 & 0.029 & 0.035 \\
\cline { 2 - 8 } & 0.5 & 0.051 & 0.037 & 0.026 & 0.023 & 0.025 & 0.029 \\
\hline
\end{tabular}

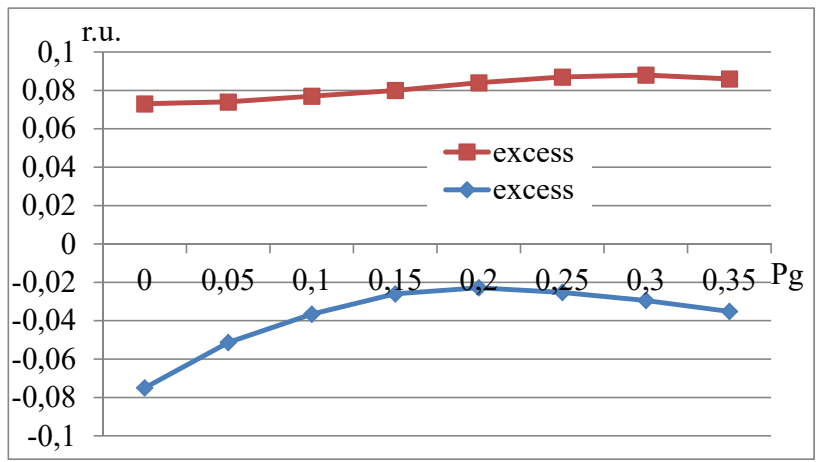

Fig. 6 . Average values of power imbalances $\left(k_{1}=k_{2}=0.5\right)$

As the example of parity capacities of WPP and SPP shows (Tables 1-3), the distinctions at different algorithms are within the variability of values and the distinction between average values of calculated parameters is at the level of several percent. In particular, the energy loss index (positive power imbalance) under condition (8) is practically independent of the unit power of the generator.

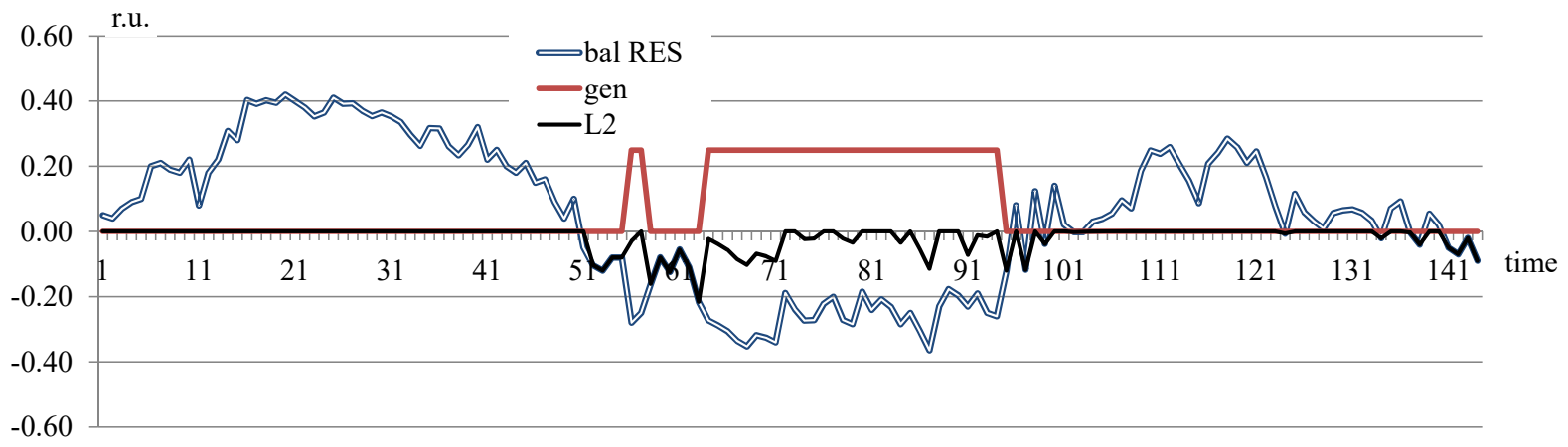

Fig. 5. Example of power balancing, daily forecast 
In addition to such an indicator as load loss which is important for normal operation of the regulating generator, there are number and frequency of on-off cycles which significantly affects the quality of its operation. When considering the sequence of the generator power values $P_{G}\left(t_{\mathrm{i}}\right)$, we are talking about the number of series being a group of significant values equal to $P_{g}$ or zero each. As the simulation shows, the number of changes $n_{g}$ in the generator operating mode decreases (Table 4) with an increase in the generator power or application of more stringent restrictions.

Table 4 Average number of generator actuations $\left(n_{g}\right)$, daily forecast

\begin{tabular}{|c|c|c|c|c|c|c|c|}
\hline \multirow{2}{*}{$k_{1}$} & $k_{2}$ & 0.05 & 0.1 & 0.15 & 0.2 & 0.25 & 0.3 \\
\hline \multirow{2}{*}{1} & 1 & 10.3 & 9.0 & 6.8 & 4.8 & 2.9 & 1.8 \\
\cline { 2 - 8 } & 0.5 & 13.2 & 12.2 & 10.4 & 8.1 & 6.0 & 3.8 \\
\hline 0.5 & 0.5 & 10.9 & 10.3 & 9.8 & 9.0 & 8.0 & 6.8 \\
\hline
\end{tabular}

As can be seen from Table 4, the number of cycles decreases substantially faster with an increase in the generator power if more stringent restrictions are applied to its actuation. At low values of the generator power, the number of actuations is less dependent on the limitations and is determined mainly by the behavior of renewable energy.

\section{Discussion of the results obtained in modeling the process of power balancing}

In contrast to [2-15], the algorithm of determining the imbalance (Fig. 2) makes it possible not only to obtain average or integrated values but also the probability distribution density. This is important for assessing the reliability of energy supply or associated risks.

Another advantage of the proposed approach consists in the ability to vary conditions of the generator on/off by changing the settings $k_{1}, k_{2}$ in (7) or (8) in contrast to previous studies. Besides, the general formulation of the optimization problem (3), (4) makes it possible to use the methods of optimal generator control to select the law of its control if its power is adjustable.

The presented results reflect qualitative dependences. Quantitative indicators can differ under other initial conditions. The calculations were performed for the following conditions:

- average loading level: 0.6 r.u.;

- nominal capacities of WPP and SPP: 1 r.u.;

- installed capacity utilization factors (ICUF) of RES (2): 0.41 and 0.19 , respectively.

This corresponds to the high RES potential typical in particular for the Black Sea northern coast. Actual values of ICUF vary during the year in the range of $0.25-0.44$ for WPP and 0.07-0.24 for SPP. Obviously, estimates of the energy balance parameters will slightly differ in each season which limits the direct applicability of the obtained results. The same applies to the ratio of installed capacities. However, simulation of different situations (with other numerical parameters) indicates the presence of analogies in the behavior of the energy balance in the system. Therefore, the proposed calculation method (algorithm in Fig. 2), in contrast to those used in other studies, has a greater unification.
Note that the data in Tables 1-4 were obtained by averaging over several hundred instantiations, however, individual instantiations may scatter substantially relative to the average value. In particular, the standard deviations (meansquare deviations) of the described parameters have values commensurate with the average values. Therefore, the probable statistical error of accepted estimates can be determined by proceeding from assumptions on the nature of deviations distribution. In particular, quantiles of random imbalance values corresponding to given reliability can be used for normal distribution.

The obtained estimates are based on mathematical expectations of the studied quantities, so, they relate to the averaged values over a significant time interval as in most of the considered studies. However, this study method features modeling of random processes which is based on their decomposition and separation of predicted and purely random components (11). This conception, in contrast to existing approaches to forecasting, makes it possible to separate the components of uncertainty and assess the impact of forecast accuracy on the range of imbalances and the corresponding need for the power of the standby generator in any system.

As the study has shown, real processes of energy generation in a hybrid system are of high volatility (for example, Table $2, \sigma_{2}$ value). That is why the averaged estimates are characterized by a high variance of values. Thus, individual instantiations or states of power balancing can differ significantly from the average values: the range of possible values is within $\pm \sigma_{2}$ at normal distribution. These shortcomings can be partially eliminated by accompanying the recommended parameters with a probability assessment which will identify possible risks in the power system operation. The quantiles of the distribution of the desired random variable of the form (9) or (10), e.g. the energy imbalance (12) will serve as an estimate of the probability (of the result of reliability).

These additional capabilities of the proposed method of modeling and calculation of energy balance have practical application in pre-selection of generating capacities in a project of constructing a power supply system as well as in assessing the optimal capacity configuration and development of ways to reliability improvement by selecting additional generation means and settings of their actuation.

Improvement of calculation accuracy and reliability of recommendations requires taking into account larger volumes of statistical data on regional climatic conditions and the peculiarities of energy consumption. It is also possible to improve the methods of solving the optimization problem (the method of the response surface was used in this study). As for the actual configuration of the power system, the method of radical improvement of the energy balance involves the introduction of energy accumulation means. The availability of an accumulator makes it possible to reduce the time of the generator operation while maintaining the level of reliability of the power supply, i.e. to improve economic and environmental components. The joint operation of the accumulator and the standby generator is the subject of further studies in this area.

\section{Conclusions}

1. To assess the possibilities of balancing the hybrid power systems, the problem of power optimization was formulated which involves the choice of share of power of wind and 
solar power plants and power and conditions of the standby generator actuation. Optimization by power indicators or energy balance is possible. The balance state was represented by a series of consecutive values varying during the day with an interval of 10 minutes. The practical solution of the problem involves simulation of random processes inherent in wind and solar power engineering as well as energy consumption regimes. For the universality of estimates, all values were taken in relative units while the nominal load capacity was taken as a unit.

2. To assess the standby generator impact on power balance, several possible options were proposed according to which the generator is turned on and off. These options involve a constant negative balance, i. e. insufficient generation power. The imbalance magnitude, duration (number of time intervals), and dynamics of change at adjacent time intervals were taken into account.

3. Current capacity of wind and solar power plants was modeled as a sum of the conventional component (trend lines), the projected daily average value (if any), and current random changes (fluctuations). A similar approach is also acceptable for modeling the load behavior, i. e. electricity consumption. The model parameters were calculated in accordance with the data of long-term observations of climatic factors at existing RES facilities and the typical nature of consumption. The procedure of calculating these parameters was proposed by the authors in previous studies.

4. Mathematical expectations for a random sample of daily instantiations by the Monte Carlo method were used as estimates of the sought parameters. The normalized mean square deviation was used as an indicator of statistical error. For example, achievement of such a deviation of not more than 0.01 relative units (r. u.) requires about a thousand instantiations. For less stringent requirements (for example, up to 0.05), 200-300 daily instantiations are enough. The obtained estimates can be considered statistically capable, given the asymptotic convergence of the results. In this case, optimal values of the standby generator power at which imbalance is minimal are within $0.1-0.3 \mathrm{r}$. u. Further increase in the generator power does not lead to a decrease in load losses. At lower power, the on-off frequency increases as well in addition to increased losses.

Daily forecasting and planning of operating modes as well as achievable accuracy of forecasting the RES indicators significantly affect the ability to ensure the desired energy balance. The use of the model of daily cyclicity seems appropriate, as many indicators depend on the time of day. It is really possible to provide daily averages but the current indicators are somewhat volatile. Therefore, practical application of the proposed approach involves a detailed assessment and consideration of local conditions, regional ones in terms of RES potential, and concretized in terms of the energy system capabilities. Observance of these conditions enables obtaining rather reliable estimates which are confirmed by comparison of the model and actual indicators for a number of RES facilities operating in Ukraine.

\section{Acknowledgments}

The article was prepared in the framework of the projects of scientific and technical works of the National Academy of Sciences of Ukraine: "Complex-C", "Complex-3" (KPKVK 6541030).

\section{References}

1. Kuznietsov, M., Melnyk, O. (2020). The influence of instability consumption on the hybrid energy system balance. Vidnovluvana Energetika, 2 (61), 8-17. doi: https://doi.org/10.36296/1819-8058.2020.2(61).8-17

2. Negi, S., Mathew, L. (2014). Hybrid Renewable Energy System: A Review. International Journal of Electronic and Electrical Engineering, 7 (5), 535-542. Available at: https://www.ripublication.com/irph/ijeee_spl/ijeeev7n5_15.pdf

3. Baba Kyari, I., Ya'u Muhammad, J. (2019). Hybrid Renewable Energy Systems for Electrification: A Review. Science Journal of Circuits, Systems and Signal Processing, 8 (2), 32. doi: https://doi.org/10.11648/j.cssp.20190802.11

4. Raza, M. Q., Nadarajah, M., Hung, D. Q., Baharudin, Z. (2017). An intelligent hybrid short-term load forecasting model for smart power grids. Sustainable Cities and Society, 31, 264-275. doi: https://doi.org/10.1016/j.scs.2016.12.006

5. Hsu, C.-C., Chen, C.-Y. (2003). Regional load forecasting in Taiwan--applications of artificial neural networks. Energy Conversion and Management, 44 (12), 1941-1949. doi: https://doi.org/10.1016/s0196-8904(02)00225-x

6. Xia, C., Wang, J., McMenemy, K. (2010). Short, medium and long term load forecasting model and virtual load forecaster based on radial basis function neural networks. International Journal of Electrical Power \& Energy Systems, 32 (7), 743-750. doi: https:// doi.org/10.1016/j.ijepes.2010.01.009

7. Khwaja, A. S., Naeem, M., Anpalagan, A., Venetsanopoulos, A., Venkatesh, B. (2015). Improved short-term load forecasting using bagged neural networks. Electric Power Systems Research, 125, 109-115. doi: https://doi.org/10.1016/j.epsr.2015.03.027

8. Rehman, S., El-Amin, I. (2015). Study of a Solar Pv/Wind/Diesel Hybrid Power System for a Remotely Located Population near Arar, Saudi Arabia. Energy Exploration \& Exploitation, 33 (4), 591-620. doi: https://doi.org/10.1260/0144-5987.33.4.591

9. Spiru, P., Lizica-Simona, P. (2018). Technical and economical analysis of a PV/wind/diesel hybrid power system for a remote area. Energy Procedia, 147, 343-350. doi: https://doi.org/10.1016/j.egypro.2018.07.102

10. Akram, M. W., Yusuf, S. S. (2021). An efficient solar-diesel hybrid power generation system for Maheshkhali Island of Bangladesh. Proceedings of the 13th International Conference on Mechanical Engineering (ICME2019). doi: https://doi.org/10.1063/5.0037473

11. Hadjipaschalis, I., Poullikkas, A., Efthimiou, V. (2009). Overview of current and future energy storage technologies for electric power applications. Renewable and Sustainable Energy Reviews, 13 (6-7), 1513-1522. doi: https://doi.org/10.1016/j.rser.2008.09.028

12. Djelailia, O., Kelaiaia, M. S., Labar, H., Necaibia, S., Merad, F. (2019). Energy hybridization photovoltaic/diesel generator/pump storage hydroelectric management based on online optimal fuel consumption per kWh. Sustainable Cities and Society, 44, 1-15. doi: https://doi.org/10.1016/j.scs.2018.09.037 
13. Khan, M. J., Yadav, A. K., Mathew, L. (2017). Techno economic feasibility analysis of different combinations of PV-Wind-DieselBattery hybrid system for telecommunication applications in different cities of Punjab, India. Renewable and Sustainable Energy Reviews, 76, 577-607. doi: https://doi.org/10.1016/j.rser.2017.03.076

14. Haghighat Mamaghani, A., Avella Escandon, S. A., Najafi, B., Shirazi, A., Rinaldi, F. (2016). Techno-economic feasibility of photovoltaic, wind, diesel and hybrid electrification systems for off-grid rural electrification in Colombia. Renewable Energy, 97, 293-305. doi: https://doi.org/10.1016/j.renene.2016.05.086

15. Suchitra, D., Utthra, R., Jegatheesan, R., Tushar, B. (2013). Optimization of a PV-Diesel hybrid Stand-Alone System using MultiObjective Genetic Algorithm. Emerging Research in Management \&Technology, 2 (5), 68-76.

16. Zhang, J., Li, H., Chen, D., Xu, B., Mahmud, M. A. (2021). Flexibility assessment of a hybrid power system: Hydroelectric units in balancing the injection of wind power. Renewable Energy, 171, 1313-1326. doi: https://doi.org/10.1016/j.renene.2021.02.122

17. Olsson, M., Perninge, M., Söder, L. (2010). Modeling real-time balancing power demands in wind power systems using stochastic differential equations. Electric Power Systems Research, 80 (8), 966-974. doi: https://doi.org/10.1016/j.epsr.2010.01.004

18. Bendat, J. S., Piersol, A. G. (2010). Random data: analysis and measurement procedures. Wiley. doi: https:// doi.org/10.1002/9781118032428

19. Lysenko, O., Kuznetsov, M., Chebanov, A., Adamova, S. (2019). Hybrid Power System Stochastic Optimization. Modern Development Paths of Agricultural Production, 385-394. doi: https://doi.org/10.1007/978-3-030-14918-5_40

20. Kuznetsov, N., Lysenko, O. (2017). Statistical analysis of energy indices of solar radiation (Based on the data of Tokmak Solar Power Station). Problemele energeticii regionale, 2 (34), 140-148. Available at: http://elar.tsatu.edu.ua/bitstream/ 123456789/5052/1/15_02_34_2017.pdf 